\title{
Improving the passive tactile digit span
}

\author{
MORTON A. HELLER \\ Winston-Salem State University, Winston-Salem, North Carolina
}

\begin{abstract}
Two experiments evaluated the effect of interstimulus interval (ISI) on recall of digit sequences printed on the palms. Digits were printed with ISIs of 1 or $5 \mathrm{sec}$. Half of the patterns were drawn on the left palm and half on the right. High levels of accuracy were obtained in both experiments with the 5-sec rate. The passive tactile digit span was nearly seven at the slower rate, but much less at 1 -sec ISIs.
\end{abstract}

Deaf-blind persons are taught to read letters printed on the palm (Print-on-Palm, POP), but little formal research has been performed to isolate the factors that may aid tactile memory (Heller, 1986b). Heller (1980) claimed that passive touch impairs retention, since the passive tactile digit span was limited to only three digits when numerals were printed over each other at the same skin locus. Furthermore, Heller (1980) argued that aftersensations degraded tactile memory for passive touch, since words were easier to read with long interstimulus intervals (ISIs) of $5 \mathrm{sec}$ than when they were printed with only $1 \mathrm{sec}$ between letters. Unfortunately, it is possible that the effect of slow presentation rates was not the dissipation of aftersensations. Instead, long ISIs may have allowed subjects to use linguistic cues as retention aids. Consequently, the present experiments examined the effect of rate on the tactile digit span. It was expected that slower rates would aid memory by allowing distracting peripheral aftersensations to fade.

\section{EXPERIMENT 1}

\section{Method}

Subjects. There were 20 subjects in the first experiment; half were male and half were female.

Stimuli and Apparatus. The stimuli of Experiment 1 were random number sequences, varying in length from 2 to 6 digits. Numbers were approximately $4 \mathrm{~cm}$ high; they were drawn with the aid of a Lucite stylus with a $.16-\mathrm{cm}$ rounded tip. An upright orientation was used, and the digits were drawn at a rapid rate.

Design and Procedure. Experiment 1 took the form of a mixed-factor analysis of variance, with the factors being interstimulus interval (1 or $5 \mathrm{sec}$ ) and sequence length (2-, 3-, 4-, 5-, or 6-digit sequences), with repeated measures on sequence length. Subjects were initially exposed to a practice run that consisted of the 10 digits two times each, presented in a random sequence, with feedback for an incorrect identification. The aim of this practice was to familiarize subjects with the method of writing the patterns and to ensure normal tactile perception. All subjects scored at least $80 \%$ correct (see Critchley, 1953). Subsequently, independent groups of subjects were exposed to number sequences with ISIs of either 1 or $5 \mathrm{sec}$.

The subjects were first exposed to 10 two-digit sequences and then to blocks of longer and longer sequences. Spatial separation was em-

This research was supported by NIH MBRS Grant 2 SO6 RR-0804017. I want to thank Icca Vonja Jones, Johnsie Miller, and Bernardine Mitchell for help with data collection and analysis. Address reprint requests to M. A. Heller, Department of Psychology, Winston-Salem State University, Winston-Salem, NC 27110. ployed to minimize distracting aftersensations (see Heller, 1986b). The first digit of two-digit sequences was printed on the left palm and the second was printed on the right palm. Half of the numbers were written on the left palm and half on the right for even numbers, with the beginning half drawn first on the left. The extra digit was always drawn on the left palm for odd numbers. Observers were fully informed about the procedure prior to the retention task. They were told where to expect the numbers, and they were informed about orientation and sequence length. However, feedback was not given as individuals attempted recall.

\section{Results and Discussion}

Tactile memory was far better when sequences were presented at a slower rate. Table 1 shows the proportion of sequences recalled correctly at 1 -sec and 5-sec ISIs. Performance was very good for long sequences at the 5sec ISI. Data analysis showed a significant effect of rate $[F(1,18)=16.3, p<.001]$, a robust effect of sequence length $[F(4,72)=39.9, p<.001]$, and a significant interaction $[F(4,72)=4.3, p<.01]$. The effect of ISI appeared to be larger for longer sequences. Moreover, the simple main effect of sequence length was significant at 1 -sec ISIs $[F(4,72)=32.1, p<.01]$ and at 5-sec ISIs $[F(4,72)=12.1, p<.01]$. A Newman-Keuls test was performed on mean number of sequences correct (with the data collapsed over ISI) and indicated that recall was worse for longer sequences ( $p<.05$ for all comparisons) and that two- and three-digit sequences were equally easy to remember $(p>.05)$.

The results of the present experiment suggest that the tactile digit span is at least six when spatial separation is employed and slow rates of presentation are used. To further quantify the normal span, an additional 10 subjects attempted serial recall of seven-digit sequences at either

Table 1

Effect of Interstimulus Interval (ISI) and Sequence Length on Mean Number of Correct Digit Sequences

\begin{tabular}{llllll}
\hline & \multicolumn{5}{c}{ Sequence Length } \\
\cline { 2 - 6 } ISI & 2 & 3 & 4 & 5 & 6 \\
\hline 1 sec & & & & & \\
Mean & 7.8 & 8.8 & 7.0 & 4.8 & 3.3 \\
SD & 1.6 & 1.9 & 2.1 & 2.8 & 2.0 \\
5 sec & & & & & \\
Mean & 9.7 & 9.8 & 9.0 & 8.8 & 6.4 \\
SD & 0.5 & 0.6 & 1.1 & 1.1 & 2.2 \\
\hline
\end{tabular}

Note-A perfect score $=10 . S D=$ Standard deviation. 
1-sec or 5-sec ISIs. The procedure was similar to that of Experiment 1, and ISI was balanced. The mean number of sequences correct was 4.9 for seven-digit sequences at the slow rate and only 2.2 at $1-\mathrm{sec}$ ISIs. The effect of rate was highly significant $[t(9)=3.6, p<.01]$.

\section{EXPERIMENT 2}

Experiment 2 was performed to test the generality of the effect of rate on retention of digit sequences. The observers in Experiment 2 attempted serial recall of digits printed on the two palms, but the method involved obtaining a span by more usual methods (e.g., Woodworth \& Schlosberg, 1954).

\section{Method}

Subjects. There were 20 subjects in Experiment 2. Half of the subjects were male and half were female.

Procedure. The procedure of Experiment 2 was similar to the twopalm method of Experiment 1, except that a traditional digit span was determined. Subjects were exposed to longer and longer sequences of digits, varying in length from three to nine digits, in three blocks of trials. Independent groups of subjects had ISIs of 1 or $5 \mathrm{sec}$.

\section{Results and Discussion}

The mean longest correct sequence was taken as an estimate of each subject's digit span. Although this method may sometimes overestimate the span, it was deemed necessary in view of the variability shown by subjects from trial to trial. The mean span estimate for the 1-sec rate was $4.3(S D=1.2)$ and $6.8(S D=1.0)$ digits for 5 -sec ISIs. The difference between the means was highly significant $[t(18)=5.1, p<.01]$. These results were consistent with those of Experiment 1.

\section{GENERAL DISCUSSION}

The results suggest that passive tactile performance can be very close to that of other senses, since recall was good at 5-sec ISIs. The effect of ISI on tactile memory cannot be explained in terms of higher cognitive functions, and suggests that aftersensations contribute to retention losses with rapid presentation rates. Furthermore, the results of the present research have clear implications for the education of the deaf- blind (see Nelipovich \& Naegele, 1985). Instruction in POP should entail slow presentation rates and separate skin loci for sequential stimuli, at least until subjects become highly practiced at the task.

The present data may bear on the theoretical distinction between active and passive touch (Gibson, 1962). Passive touch, where the observer makes no voluntary movements, may promote subjective experiences. Thus it seemed plausible that passivity may impair memory for sequentially presented tactile input (Heller, 1980). However, the results of the present study suggest that passive tactile retention need not always be deficient, if optimal conditions for presentation are used. It may be necessary to limit the range of stimuli (for POP), make this known to the subjects, and allow observers the use of two hands (see Craig, 1985; Heller, 1980, 1986b). Haptics may differ from passively generated tactile information in a couple of important ways. First, perception of sequences of information through active touch may not require the long ISIs that seem to be so beneficial for passive drawing on the skin. Second, for active touch, accurate retention of sequential input may not demand the use of separate receptor surfaces (e.g., two hands) as does passive touch. Braille words, for example, can be accurately read with one fingertip with active touch (Heller, 1986a). Moreover, the use of two hands for active exploration can sometimes even degrade performance, owing to problems with bimanual movement control (Appelle \& Countryman, 1986).

\section{REFERENCES}

ApPelle, S., \& Countryman, M. (1986). Eliminating the haptic oblique effect: Influence of scanning incongruity and prior knowledge of the standards. Perception, 15, 325-329.

Craig, J. C. (1985). Attending to two fingers: Two hands are better than one. Perception \& Psychophysics, 38, 496-511.

Critchley, M. (1953). The parietal lobes. New York: Hafner.

Gibson, J. J. (1962). Observations on active touch. Psychological Review, 69, 477-490.

Heller, M. A. (1980). Tactile retention: Reading with the skin. Perception \& Psychophysics, 27, 125-130.

HeLler, M. A. (1986a). Active and passive tactual braille recognition. Bulletin of the Psychonomic Society, 24, 201-202.

Heller, M. A. (1986b). Central and peripheral influences on tactual reading. Perception \& Psychophysics, 39, 197-204.

NelipoviCh, M., \& NAEgELE, L. (1985). The rehabilitation process for persons who are deaf and blind. Journal of Visual Impairment \& Blindness, 79, 104-109.

WoODWORTh, R. S., \& SCHLOSBerg, H. (1954). Experimental Psychology. New York: Holt, Rinehart \& Winston.

(Manuscript received for publication December 28, 1986.) 